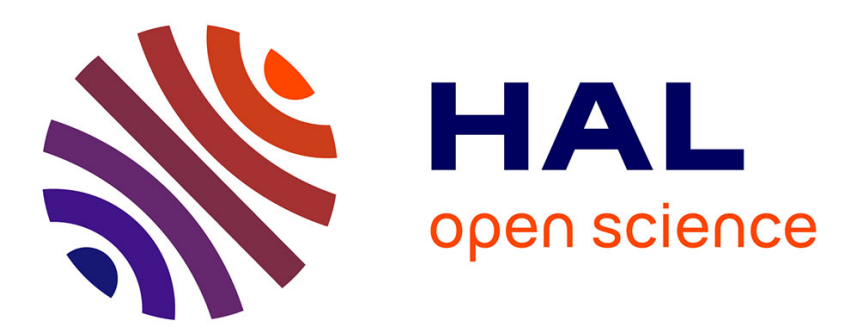

\title{
Asymptotic Performance of Linear Receivers in Network MIMO
}

Jakob Hoydis, Mari Kobayashi, Merouane Debbah

\section{To cite this version:}

Jakob Hoydis, Mari Kobayashi, Merouane Debbah. Asymptotic Performance of Linear Receivers in Network MIMO. Asilomar Conference on Signals, Systems, and Computers (Asilomar'10), Nov 2010, Pacific Grove, CA, United States. hal-00554956

\section{HAL Id: hal-00554956 \\ https://hal-centralesupelec.archives-ouvertes.fr/hal-00554956}

Submitted on 11 Jan 2011

HAL is a multi-disciplinary open access archive for the deposit and dissemination of scientific research documents, whether they are published or not. The documents may come from teaching and research institutions in France or abroad, or from public or private research centers.
L'archive ouverte pluridisciplinaire HAL, est destinée au dépôt et à la diffusion de documents scientifiques de niveau recherche, publiés ou non, émanant des établissements d'enseignement et de recherche français ou étrangers, des laboratoires publics ou privés. 


\title{
Asymptotic Performance of Linear Receivers in Network MIMO
}

\author{
(Invited Paper) \\ Jakob Hoydis, Mari Kobayashi, and Mérouane Debbah
}

\begin{abstract}
We consider the asymptotic performance of a class of linear receivers in multiple-input multiple-output (MIMO) multiple access channels (MAC). Under the assumption that the number of transmitters $K$ and the number of receive antennas $N$ grow large at the same rate and that the receiver has only an imperfect estimate of the channel matrix, we derive deterministic equivalents for the signal-to-interference-plus-noise-ratio (SINR) at the receiver output. Since we assume that the channel matrix has a variance profile, i.e., different matrix entries exhibit different variances, the results are useful for the analysis of network MIMO systems where a user terminal (UT) sees a different path loss to different cooperative base stations (BSs). Our simulation results show that the asymptotic performance predictions are accurate for even small values of $N$ and $K$.
\end{abstract}

\section{INTRODUCTION}

Multi-cell cooperation for joint transmission, detection or interference coordination, also referred to as network multipleinput multiple-output (MIMO), is a potential solution to overcome the limiting inter-cell interference in current cellular systems [1]. By exchanging user data and/or channel state information (CSI) via highspeed backhaul links, several base stations (BSs) can jointly process signals for user terminals (UTs) in multiple cells. Without any constraints on the processing complexity, availability of CSI and backhaul capacity, a network MIMO system can be represented by a MIMO multiple access channel (MAC) in the uplink or a MIMO broadcast channel in the downlink whose capacity regions are known [2], [3]. However, the aforementioned practical limitations render the analysis difficult and more realistic performance predictions of network MIMO systems are hard to obtain analytically. For a comprehensive survey of recent related results, we refer to [4].

The focus of this work is on two practical limitations of uplink network MIMO systems: processing complexity and imperfect CSI. Since joint optimal decoding might not be possible due to prohibitive complexity and full CSI is hardly available, it is of practical and theoretical interest to study the performance of linear single-user receivers assuming imperfect CSI at the BSs. Under this setting, we study the signal-to-interference-plus-noise-ratio (SINR) at the output of several linear receivers, such as the minimum-mean-squareerror (MMSE) receiver, the zero-forcing $(\mathrm{ZF})$ receiver and the matched filter (MF). The difficulty here is related to the fact that a network MIMO system is characterized by a random channel matrix whose elements have different variances, capturing the path loss differences between the links. Therefore, exact expressions of the SINR distribution or even its mean are intractable to obtain. This motivates us to study the large system limit with many UTs and many coordinated receive antennas and to derive deterministic approximations of the SINR. These approximations are only asymptotically tight but shown by simulations to yield accurate performance predictions for small system dimensions.

The asymptotic behavior of the SINR of linear receivers has been extensively studied in the context of CDMA and MIMO systems with different assumptions on the random matrix models describing the spreading sequences or channel matrices [5]-[14]. The authors of [5], [6] consider linear receivers for CDMA with random independent and identically distributed (i.i.d.) spreading sequences while [8] extends these results to the case of multipath fading channels and imperfect CSI. The asymptotic performance of the MMSE receiver in CDMA downlink systems with frequency-selective fading and random unitary spreading sequences is studied in [9]. Multicarrier CDMA over frequency-selective fading channels with random i.i.d. spreading sequences is considered in [10].

A significant body of works deals also with the fluctuations of the SINR. This is normally established under the form of a central limit theorem (CLT) and expressions of the asymptotic variance (or higher moments) are derived. The authors of [7] consider MMSE and ZF receivers for random CDMA with non-uniform power allocation while [11] assumes a Rayleigh flat-fading MIMO channel with right-sided correlation. In [12], the results of [7] are extended to non-Gaussian random spreading and mismatched MMSE receivers. A CLT for the mutual information of linear receivers in Rayleigh flat-fading channels is derived in [14] while [13] studies the SINRfluctuations at the output of the MMSE receiver for arbitrary flat-fading channels with a variance profile. The novelty of our work with respect to [8], [10], [13] is the consideration of a channel matrix with a variance profile and imperfect CSI. Our results coincide with those reported in [10] for perfect CSI.

Notations: For a matrix $\mathbf{X}=\left\{x_{i j}\right\}, \mathbf{x}_{k}$ denotes the $k$ th column vector of $\mathbf{X} . \mathbf{X}_{[k]}\left(\mathbf{x}_{[k]}\right)$ is the matrix (vector) $\mathbf{X}(\mathbf{x})$ with its $k$ th column (element) removed. We denote $\operatorname{tr} \mathbf{X}, \mathbf{X}^{\top}$ and $\mathrm{X}^{\mathrm{H}}$ the trace, transpose and complex conjugate transpose of $\mathbf{X} . \mathbf{I}_{N}$ is the identity matrix of size $N$ and $\operatorname{diag}\left(x_{1}, \ldots, x_{N}\right)$ is a diagonal matrix with elements $x_{i}$. We use $\mathrm{x} \sim \mathcal{C N}(\mathbf{m}, \mathbf{R})$ to state that the vector $\mathbf{x}$ has a circular symmetric complex Gaussian distribution with mean $\mathbf{m}$ and covariance matrix $\mathbf{R}$. Let $a_{N}$ and $b_{N}$ denote a pair of infinite sequences of random variables. We write $a_{N} \asymp b_{N}$, iff $a_{N}-b_{N} \stackrel{\text { a.s. }}{\longrightarrow} 0$ for $N \rightarrow \infty$, where $\stackrel{\text { a.s. }}{\longrightarrow}$ denotes almost sure convergence. 


\section{System Model}

We consider a MIMO MAC from $K$ single-antenna UTs to a receiver equipped with $N$ antennas. The receiver could be either a single BS or several connected BSs with a total number of $N$ antennas. The received signal vector $\mathbf{y} \in \mathbb{C}^{N}$ at a given time instant is modeled as

$$
\mathbf{y}=\mathbf{H x}+\mathbf{n}
$$

with transmit vector $\mathbf{x}=\left(x_{1}, \ldots, x_{K}\right)^{\top} \sim \mathcal{C N}\left(\mathbf{0}, P \mathbf{I}_{K}\right)$ and noise vector $\mathbf{n} \sim \mathcal{C N}\left(\mathbf{0}, \sigma^{2} \mathbf{I}_{N}\right)$. Denote $\mathbf{H}=\left\{h_{i j}\right\} \in \mathbb{C}^{N \times K}$ the channel matrix whose entries are given as $(1 \leq i \leq$ $N, 1 \leq j \leq K)$

$$
h_{i j}=\sqrt{\frac{v_{i j}}{K}} w_{i j}
$$

where $w_{i j}$ are i.i.d. complex random variables, satisfying $\mathbb{E}\left[w_{i j}\right]=0, \mathbb{E}\left[\left|w_{i j}\right|^{2}\right]=1, \mathbb{E}\left[w_{i j}^{2}\right]=0$ and $\mathbb{E}\left[\left|w_{i j}\right|^{12}\right]<\infty$, and we denote $\mathbf{V}=\left\{v_{i j}\right\} \in \mathbb{R}_{+}^{N \times K}$ the variance profile of the channel matrix $\mathbf{H}$. The transmit signal-to-noise-ratio (SNR) of each UT is defined as $\rho=P / \sigma^{2}$. We assume in the sequel that the receiver has only an estimate $\hat{\mathbf{H}}=\left\{\hat{h}_{i j}\right\} \in \mathbb{C}^{N \times K}$ of the channel matrix $\mathbf{H}$ which satisfies

$$
\mathbf{H}=\hat{\mathbf{H}}+\tilde{\mathbf{H}}
$$

where $\tilde{\mathbf{H}}=\left\{\tilde{h}_{i j}\right\} \in \mathbb{C}^{N \times K}$ denotes the estimation error. We further assume that the estimate and the estimation error are mutually independent and given as $\hat{h}_{i j}=\sqrt{\hat{v}_{i j} / K} w_{i j}^{\prime}$ and $\tilde{h}_{i j}=\sqrt{\tilde{v}_{i j} / K} w_{i j}^{\prime \prime}$, respectively, where $w_{i j}^{\prime}$ and $w_{i j}^{\prime \prime}$ satisfy the same conditions as $w_{i j}$. Denote $\hat{\mathbf{V}}=\left\{\hat{v}_{i j}\right\} \in \mathbb{R}_{+}^{N \times K}$ the variance profile of the matrix $\hat{\mathbf{H}}$ and define the following diagonal matrices $(1 \leq j \leq K)$

$$
\begin{aligned}
& \mathbf{D}_{j}=\operatorname{diag}\left(v_{1 j}, \ldots, v_{N j}\right) \\
& \hat{\mathbf{D}}_{j}=\operatorname{diag}\left(\hat{v}_{1 j}, \ldots, \hat{v}_{N j}\right) \\
& \tilde{\mathbf{D}}_{j}=\operatorname{diag}\left(\tilde{v}_{1 j}, \ldots, \tilde{v}_{N j}\right) .
\end{aligned}
$$

In this correspondence, we consider several linear receive filters $\mathrm{g}_{k} \in \mathbb{C}^{N}$, namely the MF receiver, the ZF receiver or decorrelator, and the MMSE receiver, defined respectively by [15]:

$$
\begin{aligned}
\mathbf{g}_{k}^{\mathrm{MF}} & \triangleq \hat{\mathbf{h}}_{k} \\
\mathbf{g}_{k}^{\mathrm{ZF}} & \triangleq \hat{\mathbf{\Pi}}_{k} \hat{\mathbf{h}}_{k} \\
\mathbf{g}_{k}^{\mathrm{MMSE}} & \triangleq\left(\hat{\mathbf{H}}_{[k]} \hat{\mathbf{H}}_{[k]}^{\mathrm{H}}+\tilde{\mathbf{Z}}+\frac{1}{\rho} \mathbf{I}_{N}\right)^{-1} \hat{\mathbf{h}}_{k}
\end{aligned}
$$

where

$$
\hat{\mathbf{\Pi}}_{k} \triangleq \mathbf{I}_{N}-\hat{\mathbf{H}}_{[k]}\left(\hat{\mathbf{H}}_{[k]}^{\mathrm{H}} \hat{\mathbf{H}}_{[k]}\right)^{-1} \hat{\mathbf{H}}_{[k]}^{\mathrm{H}}
$$

is the projection onto the sub-space orthogonal to the columnspace of the matrix $\hat{\mathbf{H}}_{[k]}$ and $\tilde{\mathbf{Z}} \in \mathbb{R}_{+}^{N \times N}$ is given as

$$
\tilde{\mathbf{Z}} \triangleq \mathbb{E}\left[\tilde{\mathbf{H}} \tilde{\mathbf{H}}^{\mathrm{H}}\right]=\frac{1}{K} \sum_{j=1}^{K} \tilde{\mathbf{D}}_{j} .
$$

The receiver tries to decode the message $x_{k}$ of UT $k$ based on the scalar observation

$$
\mathbf{g}_{k}^{\mathrm{H}} \mathbf{y}=\mathbf{g}_{k}^{\mathrm{H}} \hat{\mathbf{h}}_{k} x_{k}+\mathbf{g}_{k}^{\mathrm{H}}\left(\mathbf{H}_{[k]} \mathbf{x}_{[k]}+\tilde{\mathbf{h}}_{k} x_{k}+\mathbf{n}\right) .
$$

The corresponding received SINR $\gamma_{k}$ of UT $k$ reads

$$
\gamma_{k}=\frac{\left|\mathbf{g}_{k}^{\mathrm{H}} \hat{\mathbf{h}}_{k}\right|^{2}}{\mathbf{g}_{k}^{\mathrm{H}}\left(\mathbf{H}_{[k]} \mathbf{H}_{[k]}^{\mathrm{H}}+\tilde{\mathbf{h}}_{k} \tilde{\mathbf{h}}_{k}^{\mathrm{H}}+\frac{1}{\rho} \mathbf{I}_{N}\right) \mathbf{g}_{k}}
$$

with the associated instantaneous rate $R_{k}$ defined as

$$
R_{k}=\log _{2}\left(1+\gamma_{k}\right) \quad[\mathrm{bits} / \mathrm{s} / \mathrm{Hz}] .
$$

Remark 2.1: The MMSE receiver $\mathbf{g}_{k}^{\mathrm{MMSE}}$ is based on the assumption that the estimation error $\tilde{\mathbf{h}}_{k} x_{k}$ and the useful signal $\hat{\mathbf{h}}_{k} x_{k}$ are independent. Although this is not the case for finite dimensions, it follows from Lemma 4 that both terms are asymptotically independent, i.e., $x_{k}^{*} \hat{\mathbf{h}}_{k}^{\mathrm{H}} \tilde{\mathbf{h}}_{k} x_{k} \stackrel{\text { a.s. }}{\longrightarrow} 0$. Note also that the MMSE receiver requires a perfect knowledge of the variance of the estimation error to compute the matrix $\tilde{\mathbf{Z}}$.

\section{Asymptotic AnALYsis}

In this section, we will derive deterministic equivalents $\bar{\gamma}_{k}$ of the SINR $\gamma_{k}$ of UT $k$. This means that, as $N$ and $K$ grow infinitely large at the same rate, $\bar{\gamma}_{k}$ approximates $\gamma_{k}$ arbitrarily close while being independent of the actual channel realization H. More precisely, the notation $N \rightarrow \infty$ will refer in the sequel to the following condition on $N$ and $K$ :

$$
0<\liminf \frac{N}{K} \leq \limsup \frac{N}{K}<\infty .
$$

Under this assumption, we will show that

$$
\gamma_{k}-\bar{\gamma}_{k} \underset{N \rightarrow \infty}{\stackrel{\text { a.s. }}{\longrightarrow}} 0
$$

or in shorter notation: $\gamma_{k} \asymp \bar{\gamma}_{k}$. We will subsequently consider the MMSE, MF and ZF receiver. Note that the almost sure convergence of the SINR implies also that

$$
R_{k}-\log _{2}\left(1+\bar{\gamma}_{k}\right) \underset{N \rightarrow \infty}{\stackrel{\text { a.s. }}{\longrightarrow}} 0 .
$$

\section{A. Main results}

In this section, we state our main results. The proofs are provided in Section III-B while necessary lemmas and theorems are given in the Appendix. Our first result is a deterministic equivalent of the SINR of the MMSE receiver:

Theorem 1 (MMSE receiver): Let $\gamma_{k}^{\mathrm{MMSE}}$ be the SINR of UT $k$ at the output of the receive filter $\mathbf{g}_{k}^{\text {MMSE }}$. Then $\gamma_{k}^{\text {MMSE }} \asymp$ $\bar{\gamma}_{k}^{\mathrm{MMSE}}$, where

$$
\bar{\gamma}_{k}^{\mathrm{MMSE}}=\frac{1}{K} \operatorname{tr} \hat{\mathbf{D}}_{k} \mathbf{T}(-1 / \rho, \hat{\mathbf{V}}, \tilde{\mathbf{Z}})
$$

with matrix $\mathbf{T}(-1 / \rho, \hat{\mathbf{V}}, \tilde{\mathbf{Z}})$ given by Theorem 4 .

We obtain the following result for the MF receiver: 
Theorem 2 (Matched filter): Let $\gamma_{k}^{\mathrm{MF}}$ be the SINR of UT $k$ at the output of the receive filter $\mathbf{g}_{k}^{\mathrm{MF}}$, then $\gamma_{k}^{\mathrm{MF}} \asymp \bar{\gamma}_{k}^{\mathrm{MF}}$, where

$$
\bar{\gamma}_{k}^{\mathrm{MF}}=\frac{\left(\frac{1}{K} \operatorname{tr} \hat{\mathbf{D}}_{k}\right)^{2}}{\frac{1}{\rho K} \operatorname{tr} \hat{\mathbf{D}}_{k}+\frac{1}{K^{2}} \operatorname{tr} \hat{\mathbf{D}}_{k}\left(\sum_{j=1}^{K} \mathbf{D}_{j}\right)} .
$$

Our last result is a deterministic equivalent of the SINR at the output of the decorrelator:

Theorem 3 (ZF receiver): Let $\gamma_{k}^{\mathrm{ZF}}$ be the SINR of UT $k$ at the output of the receive filter $\mathbf{g}_{k}^{\mathrm{ZF}}$. Assume $\lim \inf \frac{N}{K}>1$. Then $\gamma_{k}^{\mathrm{ZF}} \asymp \bar{\gamma}_{k}^{\mathrm{ZF}}$, where

$$
\bar{\gamma}_{k}^{\mathrm{ZF}}=\frac{r_{k}^{2}}{\frac{1}{\rho} r_{k}+\frac{1}{K} \operatorname{tr} \tilde{\mathbf{Z}} \mathbf{S}_{k}}
$$

with

$$
\begin{aligned}
r_{k} & =\frac{1}{K} \operatorname{tr} \hat{\mathbf{D}}_{k}\left(\mathbf{I}_{N}-\mathbf{R}(0, \hat{\mathbf{V}})\right) \\
\mathbf{S}_{k} & =\hat{\mathbf{D}}_{k}\left(\mathbf{I}_{N}-2 \mathbf{R}(0, \hat{\mathbf{V}})\right)+\mathbf{Q}\left(\hat{\mathbf{V}}, \hat{\mathbf{D}}_{k}\right)
\end{aligned}
$$

where the matrices $\mathbf{R}(0, \hat{\mathbf{V}})$ and $\mathbf{Q}\left(\hat{\mathbf{V}}, \hat{\mathbf{D}}_{k}\right)$ are given by Theorems 5 and 6 , respectively, and $\tilde{\mathbf{Z}}$ is defined in (7).

Remark 3.1: For Theorem 1 and 3, the matrix $\hat{\mathbf{V}}$ can be replaced by $\hat{\mathbf{V}}_{[k]}$ to achieve a better approximation for small values of $N$ and $K$. Similarly, for Theorem 2, the term $\frac{1}{K^{2}} \operatorname{tr} \hat{\mathbf{D}}_{k}^{2}$ can be subtracted from the denominator.

We will now study the asymptotic behavior of the deterministic equivalent SINR expressions in the low and high SNR regimes. At low SNR $(\rho \rightarrow 0)$, we get the following approximations after straight-forward computations:

$$
\bar{\gamma}_{k, \text { low }} \approx \begin{cases}\rho \frac{1}{K} \operatorname{tr} \hat{\mathbf{D}}_{k} & (\mathrm{MMSE}) \\ \rho \frac{1}{K} \operatorname{tr} \hat{\mathbf{D}}_{k} & (\mathrm{MF}) \\ \rho \frac{1}{K} \operatorname{tr} \hat{\mathbf{D}}_{k}-\frac{1}{K} \operatorname{tr} \hat{\mathbf{D}}_{k} \mathbf{R}(0, \hat{\mathbf{V}}) & (\mathrm{ZF}) .\end{cases}
$$

Clearly, MMSE and MF receiver achieve a similar performance at low SNR and outperform the ZF receiver. Moreover, the performance of all receivers is independent of the estimation error.

At high SNR $(\rho \rightarrow \infty)$, we obtain the following results:

$$
\bar{\gamma}_{k, \text { high }} \approx \begin{cases}\frac{1}{K} \operatorname{tr} \hat{\mathbf{D}}_{k} \mathbf{T}(0, \hat{\mathbf{V}}, \tilde{\mathbf{Z}}) & (\mathrm{MMSE}) \\ \frac{\left(\frac{1}{K} \operatorname{tr} \hat{\mathbf{D}}_{k}\right)^{2}}{\frac{1}{K^{2}} \operatorname{tr} \mathbf{D}_{k}\left(\sum_{j=1}^{K} \mathbf{D}_{j}\right)} & (\mathrm{MF}) \\ \frac{r_{k}^{2}}{\frac{1}{K} \operatorname{tr} \tilde{\mathbf{Z}} \mathbf{S}_{k}} & (\mathrm{ZF}) .\end{cases}
$$

Note that the SINR saturates for all receivers if the estimation error does not vanish with increasing SNR, i.e., $\tilde{\mathbf{Z}}$ has full rank. For perfect CSI, the SINR of the MMSE and ZF receiver grows without bound.

\section{B. Proofs of Theorems 1, 2, and 3}

All proofs follow in essence the same steps: First, we derive deterministic equivalents of the useful signal power (numerator of (9)) and the power of the interference and noise (denominator of (9)). Since all quantities are (almost surely) bounded, it follows then from Lemma 1 that their ratio is a deterministic equivalent of the SINR.

Proof of Theorem 1 (MMSE receiver): For brevity, we write $\mathbf{g}_{k}$ instead of $\mathbf{g}_{k}^{\text {MMSE }}$.

1) Signal Power: The deterministic equivalent of the numerator of (9) can be derived as follows:

$$
\begin{aligned}
\left|\mathbf{g}_{k}^{\mathrm{H}} \hat{\mathbf{h}}_{k}\right|^{2} & =\mathbf{g}_{k}^{\mathrm{H}} \hat{\mathbf{h}}_{k} \hat{\mathbf{h}}_{k}^{\mathrm{H}} \mathbf{g}_{k} \\
& \stackrel{(a)}{ }\left(\frac{1}{K} \operatorname{tr} \hat{\mathbf{D}}_{k}\left(\hat{\mathbf{H}}_{[k]} \hat{\mathbf{H}}_{[k]}^{\mathrm{H}}+\tilde{\mathbf{Z}}+\rho^{-1} \mathbf{I}_{N}\right)^{-1}\right)^{2} \\
& \stackrel{(b)}{ }\left(\frac{1}{K} \operatorname{tr} \hat{\mathbf{D}}_{k}\left(\hat{\mathbf{H}} \hat{\mathbf{H}}^{\mathrm{H}}+\tilde{\mathbf{Z}}+\rho^{-1} \mathbf{I}_{N}\right)^{-1}\right)^{2} \\
& \stackrel{(c)}{ }\left(\frac{1}{K} \operatorname{tr} \hat{\mathbf{D}}_{k} \mathbf{T}(-1 / \rho, \hat{\mathbf{V}}, \tilde{\mathbf{Z}})\right)^{2}
\end{aligned}
$$

where $(a)$ is obtained from a direct application of Lemma 3, $(b)$ is due to Lemma 5 and $(c)$ results from Theorem 4 together with Lemma 1 . Notice that the accuracy of the approximation for small $N, K$ can be improved by ignoring step $(b)$ and replacing $\hat{\mathbf{V}}$ in the last line by $\hat{\mathbf{V}}_{[k]}$.

2) Interference and noise power: The deterministic equivalent of the denominator of (9) can be derived by the following sequence of equations:

$$
\begin{aligned}
& \mathbf{g}_{k}^{\mathrm{H}}\left(\mathbf{H}_{[k]} \mathbf{H}_{[k]}^{\mathrm{H}}+\tilde{\mathbf{h}}_{k} \tilde{\mathbf{h}}_{k}^{\mathrm{H}}+\rho^{-1} \mathbf{I}_{N}\right) \mathbf{g}_{k} \\
&= \hat{\mathbf{h}}_{k}^{\mathrm{H}}\left(\hat{\mathbf{H}}_{[k]} \hat{\mathbf{H}}_{[k]}^{\mathrm{H}}+\tilde{\mathbf{Z}}+\rho^{-1} \mathbf{I}_{N}\right)^{-1} \hat{\mathbf{h}}_{k}-\mathbf{g}_{k}^{\mathrm{H}} \tilde{\mathbf{Z}}_{k} \\
&+\mathbf{g}_{k}^{\mathrm{H}}\left(\tilde{\mathbf{H}} \tilde{\mathbf{H}}^{\mathrm{H}}+\hat{\mathbf{H}}_{[k]} \tilde{\mathbf{H}}_{[k]}^{\mathrm{H}}+\tilde{\mathbf{H}}_{[k]} \hat{\mathbf{H}}_{[k]}^{\mathrm{H}}\right) \mathbf{g}_{k} \\
& \stackrel{(a)}{=} \frac{1}{K} \operatorname{tr} \hat{\mathbf{D}}_{k} \mathbf{T}(-1 / \rho, \hat{\mathbf{V}}, \tilde{\mathbf{Z}}) \\
&-\mathbf{g}_{k}^{\mathrm{H}} \frac{1}{K} \sum_{j=1}^{K} \tilde{\mathbf{D}}_{j} \mathbf{g}_{k}+\mathbf{g}_{k}^{\mathrm{H}} \sum_{j=1}^{K} \tilde{\mathbf{h}}_{j} \tilde{\mathbf{h}}_{j}^{\mathrm{H}} \mathbf{g}_{k} \\
& \stackrel{(b)}{=} \frac{1}{K} \operatorname{tr} \hat{\mathbf{D}}_{k} \mathbf{T}(-1 / \rho, \hat{\mathbf{V}}, \tilde{\mathbf{Z}})
\end{aligned}
$$

where $(a)$ is obtained by applying Lemmas 3 and 5 and then Theorem 4 to the first term and realizing that terms involving products of the matrices $\hat{\mathbf{H}}_{[k]}$ and $\tilde{\mathbf{H}}_{[k]}^{\mathrm{H}}$ vanish due to Lemma 4, (b) is a simple consequence of Lemma 3. Again, we can replace $\hat{\mathbf{V}}$ by $\hat{\mathbf{V}}_{[k]}$ for a higher accuracy for small $N, K$.

Since the denominator and numerator of (9) are almost surely bounded and their respective deterministic equivalent approximations are bounded over $N$ and $K$, we can apply Lemma 1 to their ratio to conclude the proof.

Proof of Theorem 2 (Matched filter): The proof follows along the same lines as the proof of Theorem 1 and will not be given in full length here. 


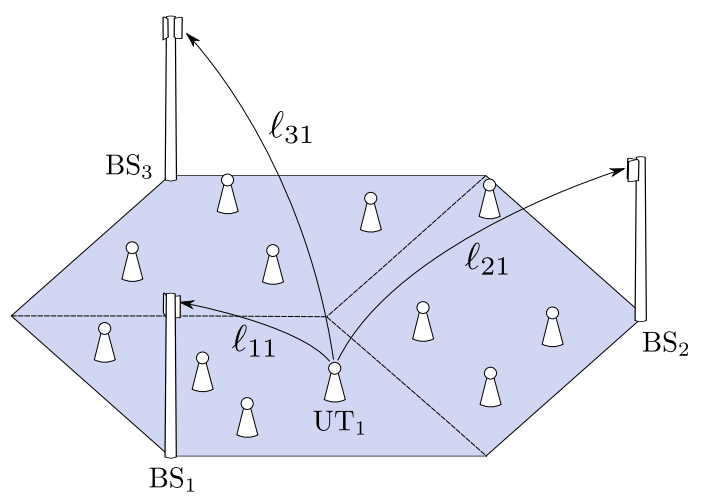

Fig. 1. Cellular example with $B=3$ cooperative BSs and $K=12$ UTs.

Proof of Theorem 3 (ZF receiver):

1) Signal Power: A deterministic equivalent of the numerator of (9) can be derived as follows:

$$
\begin{aligned}
\left|\mathbf{g}_{k}^{\mathrm{H}} \hat{\mathbf{h}}_{k}\right|^{2} & =\left|\hat{\mathbf{h}}_{k}^{\mathrm{H}} \hat{\mathbf{\Pi}}_{k} \hat{\mathbf{h}}_{k}\right|^{2} \\
& \stackrel{(a)}{ }\left(\frac{1}{K} \operatorname{tr} \hat{\mathbf{D}}_{k} \hat{\mathbf{\Pi}}_{k}\right)^{2} \\
& \stackrel{(b)}{ }\left(\frac{1}{K} \operatorname{tr} \hat{\mathbf{D}}_{k}\left(\mathbf{I}_{N}-\mathbf{R}(0, \hat{\mathbf{V}})\right)^{2}\right. \\
& =r_{k}^{2}
\end{aligned}
$$

where $(a)$ follows from Lemma 3 and $(b)$ is due to Theorem 5 and Lemma 5. Note that Lemma 5 is not valid for $z=0$. However, we claim that similar to [16], the smallest eigenvalue of $\hat{\mathbf{H}}_{k}^{\mathrm{H}} \hat{\mathbf{H}}_{k}$ is almost surely bounded away from zero by some $\epsilon>0$ for $\lim \inf \frac{N}{K}>1$, so that we can work with the almost surely nonnegative definite matrix $\hat{\mathbf{H}}_{k}^{\mathrm{H}} \hat{\mathbf{H}}_{k}-\epsilon \mathbf{I}_{K}$ instead (see proof of Theorem 5). If we do not apply Lemma 5 here, the matrix $\hat{\mathbf{V}}$ is replaced by $\hat{\mathbf{V}}_{[k]}$.

2) Interference and noise power: The deterministic equivalent of the denominator of (9) is obtained as follows:

$$
\begin{aligned}
& \mathbf{g}_{k}^{\mathrm{H}}\left(\mathbf{H}_{[k]} \mathbf{H}_{[k]}^{\mathrm{H}}+\tilde{\mathbf{h}}_{k} \tilde{\mathbf{h}}^{\mathrm{H}}+\frac{1}{\rho} \mathbf{I}_{N}\right) \mathbf{g}_{k} \\
& \stackrel{(a)}{=} \mathbf{g}_{k}^{\mathrm{H}} \tilde{\mathbf{H}} \tilde{\mathbf{H}}^{\mathrm{H}} \mathbf{g}_{k}+\frac{1}{\rho} \hat{\mathbf{h}}_{k}^{\mathrm{H}} \hat{\mathbf{\Pi}}_{k} \hat{\mathbf{h}}_{k} \\
& \stackrel{(b)}{=} \frac{1}{K^{2}} \operatorname{tr}\left(\sum_{j=1}^{K} \tilde{\mathbf{D}}_{j}\right) \hat{\mathbf{\Pi}}_{k} \hat{\mathbf{D}}_{k} \hat{\boldsymbol{\Pi}}_{k}+\frac{1}{\rho} r_{k} \\
& \stackrel{(c)}{=} \frac{1}{K} \operatorname{tr} \tilde{\mathbf{Z}} \mathbf{S}_{k}+\frac{1}{\rho} r_{k}
\end{aligned}
$$

where $(a)$ is due to $\hat{\boldsymbol{\Pi}}_{k} \hat{\mathbf{H}}_{k}=\mathbf{0}$ and $\hat{\boldsymbol{\Pi}}_{k} \hat{\boldsymbol{\Pi}}_{k}^{\mathrm{H}}=\hat{\boldsymbol{\Pi}}_{k},(b)$ follows from Lemma 3 and Theorem 5, and (c) results from Lemma 3 , Lemma 5 and Theorems 5 and 6.

\section{Numerical Results}

In order to verify the analysis in the previous sections, we consider a simple cellular system consisting of $B=3$ cooperative BSs, equipped with $M=6$ antennas each (i.e.,
$N=B M=18$ ), serving $K=12$ UTs uniformly distributed over three cell sectors. An example is shown in Fig. 1. The inverse path loss factor $\ell_{b j}$ between UT $j$ and $\mathrm{BS} b$ is given as

$$
\ell_{b j}=d_{b j}^{-3.6}
$$

where $d_{b j}$ is the distance between UT $j$ and BS $b$, normalized to the maximum distance within a cell. Since the path loss between a UT and all antennas of a BS is assumed to be the same, this results in the variance profile $\mathbf{V}$ with elements $v_{i j}=$ $\ell_{\left\lceil\frac{i}{M}\right\rceil j}$. We further assume that the variance of the channel estimate and the estimation error satisfy

$$
\hat{v}_{i j}=(1-\tau) v_{i j}, \quad \tilde{v}_{i j}=\tau v_{i j}
$$

where $\tau \in[0,1]$. We consider one random snapshot of user distributions which defines $\mathbf{V}$ and average over many different realizations of $\hat{\mathbf{H}}$ and $\tilde{\mathbf{H}}$. We consider Rayleigh fading channels, i.e., $w_{i j} \sim \mathcal{C N}(0,1)$.

Fig. 2 compares the received SINR $\gamma_{k}$ of a randomly picked UT versus the transmit SNR $\rho$ for the three different linear receivers with perfect $(\tau=0)$ and imperfect $(\tau=0.05)$ CSI. Simulation results are shown by markers, where the errorbars represent one standard deviation in each direction. The deterministic equivalent approximations $\bar{\gamma}_{k}$ are drawn by solid and dashed lines. We observe a very good approximation of the average SINR by the deterministic equivalents with a standard deviation of the instantaneous SINR of about $2.5 \mathrm{~dB}$. As expected, the performance of the MMSE and MF receiver are identical at low SNR while MMSE and ZF receiver achieve a similar performance at high SNR. Note that for imperfect CSI, both receivers are not identical at high SNR (see (5)).

Fig. 3 shows the corresponding rates $R_{k}$ versus $\rho$ and their deterministic approximations $\bar{R}_{k}=\log _{2}\left(1+\bar{\gamma}_{k}\right)$. Again, $\bar{R}_{k}$ provides a good approximation of the average rate $R_{k}$ for all three receive filters with perfect and and imperfect CSI. The standard deviation of the instantaneous rates is about $0.5 \mathrm{bits} / \mathrm{s} / \mathrm{Hz}$ in each direction.

\section{CONCLUSION}

Based on asymptotic results from random matrix theory, we have derived deterministic approximations of the SINR at the output of several linear receivers for arbitrary flat-fading channel matrices with a variance profile and imperfect CSI at the receiver. Simulations show that the asymptotic results provide tight approximations of the SINR and the associated rates for channels of rather small dimensions. Our results find applications in the context of network MIMO systems were a UT sees a different path loss to each of the cooperative BSs.

\section{APPENDIX}

Lemma 1: [17, Lemma 1] Denote $a_{N}, \bar{a}_{N}, b_{N}$ and $\bar{b}_{N}$ four infinite sequences of complex random variables indexed by $N$ and assume $a_{N} \asymp \bar{a}_{N}$ and $b_{N} \asymp \bar{b}_{N}$. If $\left|a_{N}\right|,\left|\bar{b}_{N}\right|$ and/or $\left|\bar{a}_{N}\right|,\left|b_{N}\right|$ are uniformly bounded above over $N$ (almost surely), then $a_{N} b_{N} \asymp \bar{a}_{N} \bar{b}_{N}$. Similarly, if $\left|a_{N}\right|,\left|\bar{b}_{N}\right|^{-1}$ and/or $\left|\bar{a}_{N}\right|,\left|b_{N}\right|^{-1}$ are uniformly bounded above over $N$ (almost surely), then $a_{N} / b_{N} \asymp \bar{a}_{N} / \bar{b}_{N}$. 


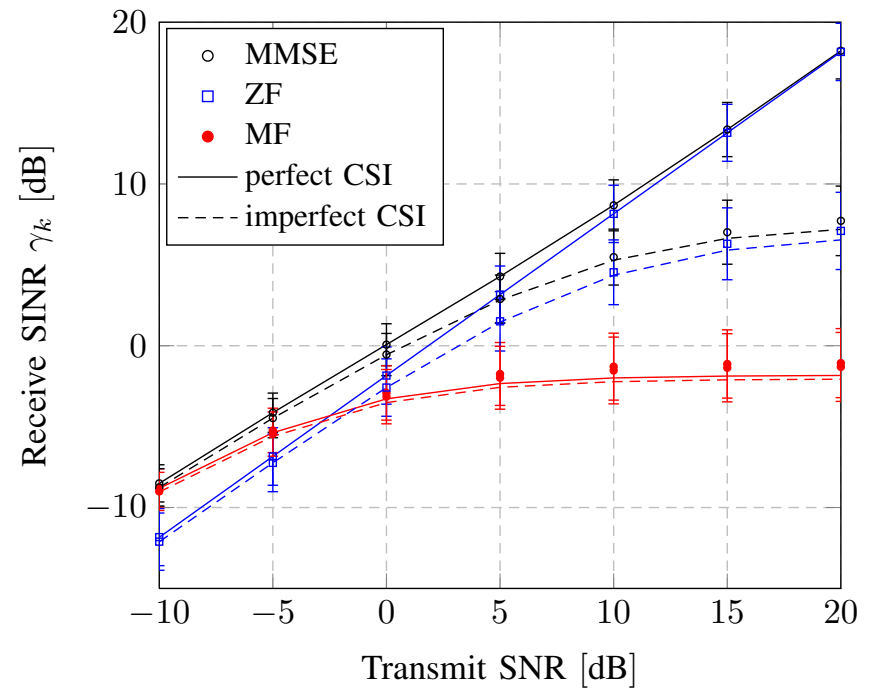

Fig. 2. SINR $\gamma_{k}$ vs $\rho$ for the MMSE, ZF and MF receiver with perfect CSI $(\tau=0)$ and imperfect CSI $(\tau=0.05)$. The deterministic approximations are drawn by solid and dashed lines, simulation results are indicated by markers. Errorbars represent one standard deviation in each direction.

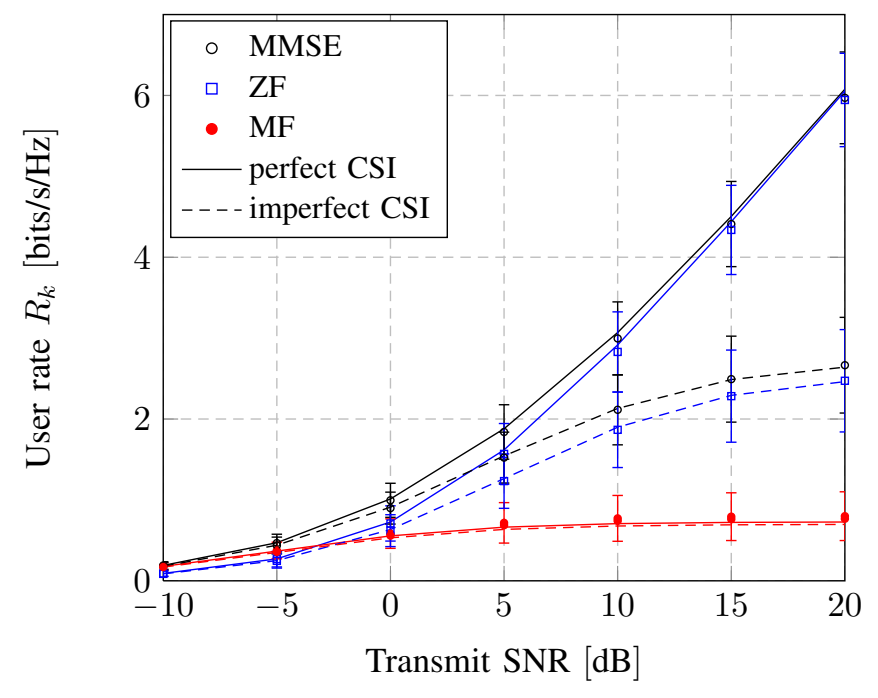

Fig. 3. User rate $R_{k}$ vs $\rho$ for the MMSE, ZF and MF receiver with perfect CSI $(\tau=0)$ and imperfect CSI $(\tau=0.05)$. The deterministic approximations are drawn by solid and dashed lines, simulation results are indicated by markers. Errorbars represent one standard deviation in each direction.

Lemma 2 (Matrix inversion lemma): [18, Eq. (2.2)] Let $\mathbf{A} \in \mathbb{C}^{N \times N}$ be Hermitian invertible. Then, for any vector $\mathbf{x} \in \mathbb{C}^{N}$ and any scalar $\tau \in \mathbb{C}$ such that $\mathbf{A}+\tau \mathbf{x x}^{\mathrm{H}}$ is invertible,

$$
\mathbf{x}^{\mathrm{H}}\left(\mathbf{A}+\tau \mathbf{x} \mathbf{x}^{\mathrm{H}}\right)^{-1}=\frac{\mathbf{x}^{\mathrm{H}} \mathbf{A}^{-1}}{1+\tau \mathbf{x}^{\mathrm{H}} \mathbf{A}^{-1} \mathbf{x}} .
$$

Lemma 3 (Trace lemma): Let $\mathbf{A} \in \mathbb{C}^{N \times N}$ be a deterministic matrix and let $\mathbf{x}=\left[x_{1}, \ldots, x_{N}\right]^{\top} \in \mathbb{C}^{N}$ be a random vector of i.i.d. entries with zero mean and variance $1 / N$, independent of A. Consider $p \geq 1, q \geq 2$ and assume that
$\mathbb{E}\left[\left|\sqrt{N} x_{1}\right|^{4 q(p-1)}\right]<\infty$. Then there exists a constant $C_{p q}$, independent of $N$ and $\mathbf{A}$, such that

$$
\mathbb{E}\left[\left|\left(\mathbf{x}^{\mathrm{H}} \mathbf{A} \mathbf{x}\right)^{p}-\left(\frac{1}{N} \operatorname{tr} \mathbf{A}\right)^{p}\right|^{q}\right] \leq \frac{C_{p q}\|\mathbf{A}\|^{p q}}{N^{\frac{q}{2}}} .
$$

This implies for $\mathbf{A}$ with uniformly bounded spectral norm

$$
\left(\mathbf{x}^{\mathrm{H}} \mathbf{A} \mathbf{x}\right)^{p}-\left(\frac{1}{N} \operatorname{tr} \mathbf{A}\right)^{p} \underset{N \rightarrow \infty}{\stackrel{\text { a.s. }}{\longrightarrow}} 0 \text {. }
$$

Proof: The case $p=1$ was already proved in [16, Lemma 2.7]. The general proof unfolds directly from the following chain of inequalities:

$$
\begin{aligned}
& \mathbb{E}\left[\left|\left(\mathbf{x}^{\mathrm{H}} \mathbf{A} \mathbf{x}\right)^{p}-\left(\frac{1}{N} \operatorname{tr} \mathbf{A}\right)^{p}\right|^{q}\right] \\
& \stackrel{(a)}{=} \mathbb{E}\left[\left.\left|\mathbf{x}^{\mathrm{H}} \mathbf{A} \mathbf{x}-\frac{1}{N} \operatorname{tr} \mathbf{A}\right|\right|^{q}\left|\sum_{k=0}^{p-1}\left(\frac{1}{N} \operatorname{tr} \mathbf{A}\right)^{p-1-k}\left(\mathbf{x}^{\mathrm{H}} \mathbf{A} \mathbf{x}\right)^{k}\right|^{q}\right] \\
& \stackrel{(b)}{\leq} \sqrt{\mathbb{E}\left[\left|\mathbf{x}^{\mathrm{H}} \mathbf{A} \mathbf{x}-\frac{1}{N} \operatorname{tr} \mathbf{A}\right|^{2 q}\right]} \\
& \quad \times \sqrt{\mathbb{E}\left[\left|\sum_{k=0}^{p-1}\left(\frac{1}{N} \operatorname{tr} \mathbf{A}\right)^{p-1-k}\left(\mathbf{x}^{\mathrm{H}} \mathbf{A} \mathbf{x}\right)^{k}\right|^{2 q}\right]} \\
& \stackrel{(c)}{\leq} \frac{C^{\prime}\|\mathbf{A}\|^{q}}{N^{\frac{q}{2}}} \sqrt{\mathbb{E}\left[p^{2 q-1} \sum_{k=0}^{p-1}\left|\frac{1}{N} \operatorname{tr} \mathbf{A}\right|^{2 q(p-1-k)}\left|\mathbf{x}^{\mathrm{H}} \mathbf{A} \mathbf{x}\right|^{2 q k}\right]} \\
& \stackrel{(\text { d) }}{\leq} \frac{C^{\prime}\|\mathbf{A}\|^{q}}{N^{\frac{q}{2}}} \sqrt{p^{2 q-1} \sum_{k=0}^{p-1}\|\mathbf{A}\|^{2 q(p-1-k)} \mathbb{E}\left[\left|\mathbf{x}^{\mathrm{H}} \mathbf{A} \mathbf{x}\right|^{2 q k}\right]} \\
& \stackrel{(e)}{\leq} \frac{C^{\prime}\|\mathbf{A}\|^{p q}}{N^{\frac{q}{2}}} \sqrt{p^{2 q-1} \sum_{k=0}^{p-1} K_{2 q k}} \\
& \leq \frac{C_{p q}\|\mathbf{A}\|^{p q}}{N^{\frac{q}{2}}}
\end{aligned}
$$

for some $C_{p q} \geq C^{\prime} \sqrt{p^{2 q-1} \sum_{k=0}^{p-1} K_{2 q k}}$, where (a) follows from $a^{n}-b^{n}=(a-b) \sum_{k=0}^{n-1} a^{n-1-k} b^{k}$, (b) follows from the Cauchy-Schwarz inequality, $(c)$ is a result of Lemma 3 with $p=1$ and Hölder's inequality, $(d)$ follows from $\frac{1}{N} \operatorname{tr} \mathbf{A} \leq$ $\|\mathbf{A}\|$, and $(e)$ results from

$$
\begin{aligned}
& \mathbb{E}\left[\left|\mathbf{x}^{\mathrm{H}} \mathbf{A} \mathbf{x}\right|^{r}\right] \\
= & \mathbb{E}\left[\sum_{\substack{i_{1}, \ldots, i_{r} \\
j_{1} \ldots j_{r}}} x_{i_{1}}^{*} \cdots x_{i_{r}}^{*} x_{j_{1}} \cdots x_{j_{r}}\right. \\
\leq & \left.K_{r} \mid \frac{1}{N} \sum_{i_{1}, j_{1}}[\mathbf{A}]_{i_{1} j_{1}}\left[\mathbf{A}_{i_{1} j_{1}}|\cdots| \frac{1}{N}\right]_{i_{2} j_{2}} \cdots[\mathbf{A}]_{i_{r-1} j_{r-1}}\left[\mathbf{A}^{\mathrm{H}}\right]_{i_{r} j_{r}}\right] \\
\leq & \left.K_{r} \| \mathbf{A}\right]_{i_{r} j_{r}} \mid
\end{aligned}
$$

where $K_{r}=\mathbb{E}\left[N^{r}\left|x_{i_{1}}\right|^{2 r}\right]<\infty$ and the last inequality follows from $\left|\frac{1}{N} \sum_{i j}[\mathbf{A}]_{i j}\right|=\left|\frac{1}{N} \mathbf{1}_{N}^{\mathrm{T}} \mathbf{A} \mathbf{1}_{N}\right| \leq\|\mathbf{A}\|$. 
Lemma 4: [19, Theorem 17] Let $\mathbf{A} \in \mathbb{C}^{N \times N}$ be a deterministic matrix with uniformly bounded spectral norm. Let $\mathbf{x} \in \mathbb{C}^{N}$ and $\mathbf{y} \in \mathbb{C}^{N}$ be two random vectors of i.i.d. entries with zero mean, variance $1 / N$ and finite fourth moment, both independent of $\mathbf{A}$. Then there exists a constant $C$, independent of $N$ and $\mathbf{A}$, such that

$$
\mathbb{E}\left[\left|\mathbf{x}^{\mathrm{H}} \mathbf{A} \mathbf{y}\right|^{4}\right] \leq \frac{C}{N^{2}} .
$$

This implies in particular

$$
\mathbf{x}^{\mathrm{H}} \mathbf{A} \mathbf{y} \underset{N \rightarrow \infty}{\stackrel{\text { a.s. }}{\longrightarrow}} 0 \quad \text { and } \quad\left|\mathbf{x}^{\mathrm{H}} \mathbf{A} \mathbf{y}\right|^{2} \underset{N \rightarrow \infty}{\stackrel{\text { a.s. }}{\longrightarrow}} 0 .
$$

Lemma 5 (Rank-1 perturbation lemma [18]): Let $z<0$, $\mathbf{A} \in \mathbb{C}^{N \times N}, \mathbf{B} \in \mathbb{C}^{N \times N}$ with B Hermitian nonnegative definite, and $\mathbf{v} \in \mathbb{C}^{N}$. Then,

$$
\left|\operatorname{tr}\left(\left(\mathbf{B}-z \mathbf{I}_{N}\right)^{-1}-\left(\mathbf{B}+\mathbf{v} \mathbf{v}^{\mathrm{H}}-z \mathbf{I}_{N}\right)^{-1}\right) \mathbf{A}\right| \leq \frac{\|\mathbf{A}\|}{|z|} .
$$

Theorem 4 (Deterministic equivalent): [20, Theorem 1 (see also [21, Lemmas 6.1 and 6.6]] Let $\mathbf{H}=\left\{h_{i j}\right\} \in \mathbb{C}^{N \times K}$ be a random matrix whose elements are given as $h_{i j}=$ $\sqrt{v_{i j} / K} w_{i j}$, where $w_{i j}$ are i.i.d., have zero mean, unit variance, and there exists $\epsilon>0$ such that $\mathbb{E}\left[\left|w_{i j}\right|^{4+\epsilon}\right]<\infty$. Denote $\mathbf{V}=\left\{v_{i j}\right\} \in \mathbb{R}_{+}^{N \times K}$ the variance profile of $\mathbf{H}$. Let $\mathbf{A} \in$ $\mathbb{C}^{N \times N}, \overline{\mathbf{A}} \in \mathbb{C}^{K \times K}, \mathbf{B} \in \mathbb{C}^{N \times N}$ and $\overline{\mathbf{B}} \in \mathbb{C}^{K \times K}$ be deterministic nonnegative hermitian matrices with bounded spectral norm and assume that $\max _{i, j} v_{i j} \leq v_{\max }<\infty$. Define the following diagonal matrices $\mathbf{D}_{j}=\operatorname{diag}\left(v_{1 j}, \ldots, v_{N j}\right), j=$ $1, \ldots, K$, and $\overline{\mathbf{D}}_{i}=\operatorname{diag}\left(v_{i 1}, \ldots, v_{i K}\right), i=1, \ldots, N$. Then, for $z \in \mathbb{C} \backslash \mathbb{R}_{+}$, the following implicit equations:

$$
\begin{aligned}
& \mathbf{T}(z, \mathbf{V}, \mathbf{A})=\left(\frac{1}{K} \sum_{j=1}^{K} \frac{\mathbf{D}_{j}}{1+\frac{1}{K} \operatorname{tr} \mathbf{D}_{j} \mathbf{T}(z, \mathbf{V}, \mathbf{A})}+\mathbf{A}-z \mathbf{I}_{N}\right)^{-1} \\
& \overline{\mathbf{T}}(z, \mathbf{V}, \overline{\mathbf{A}})=\left(\frac{1}{K} \sum_{i=1}^{N} \frac{\overline{\mathbf{D}}_{i}}{1+\frac{1}{K} \operatorname{tr} \overline{\mathbf{D}}_{i} \overline{\mathbf{T}}(z, \mathbf{V}, \overline{\mathbf{A}})}+\overline{\mathbf{A}}-z \mathbf{I}_{K}\right)^{-1}
\end{aligned}
$$

admit unique solutions $\mathbf{T}(z, \mathbf{V}, \mathbf{A}) \in \mathbb{C}^{N \times N}, \overline{\mathbf{T}}(z, \mathbf{V}, \overline{\mathbf{A}}) \in$ $\mathbb{C}^{K \times K}$, such there exist matrix-valued measures $\mu$ and $\bar{\mu}$, satisfying $\mathbf{T}(z, \mathbf{V}, \mathbf{A})=\int_{\mathbb{R}_{+}} \frac{\mu(d \lambda)}{\lambda-z}, \overline{\mathbf{T}}(z, \mathbf{V}, \overline{\mathbf{A}})=$ $\int_{\mathbb{R}_{+}} \frac{\bar{\mu}(d \lambda)}{\lambda-z}$ where $\mu\left(\mathbb{R}_{+}\right)=\mathbf{I}_{N}, \bar{\mu}\left(\mathbb{R}_{+}\right)=\mathbf{I}_{K}$. In particular, $\frac{1}{N} \operatorname{tr} \mathbf{T}(z, \mathbf{V}, \mathbf{A})$ and $\frac{1}{K} \operatorname{tr} \overline{\mathbf{T}}(z, \mathbf{V}, \overline{\mathbf{A}})$ are Stieltjes transforms of probability measures over $\mathbb{R}_{+}$.

Moreover, assume that $N, K$ grow to infinity, satisfying $0<$ $\lim \inf \frac{N}{K} \leq \limsup \frac{N}{K}<\infty$. Then, the following limits hold for any $z \in \mathbb{C} \backslash \mathbb{R}_{+}$:

$$
\begin{aligned}
& \frac{1}{K} \operatorname{tr} \mathbf{B}\left[\left(\mathbf{H H}^{\mathrm{H}}+\mathbf{A}-z \mathbf{I}_{N}\right)^{-1}-\mathbf{T}(z, \mathbf{V}, \mathbf{A})\right] \underset{N, K \rightarrow \infty}{\stackrel{\text { a.s. }}{\longrightarrow} 0} \\
& \frac{1}{K} \operatorname{tr} \overline{\mathbf{B}}\left[\left(\mathbf{H}^{\mathrm{H}} \mathbf{H}+\overline{\mathbf{A}}-z \mathbf{I}_{K}\right)^{-1}-\overline{\mathbf{T}}(z, \mathbf{V}, \overline{\mathbf{A}})\right] \underset{N, K \rightarrow \infty}{\stackrel{\text { a.s. }}{\longrightarrow}} 0 .
\end{aligned}
$$

Theorem 5: Under the assumptions of Theorem 4, let $\mathbf{C}=$ $\operatorname{diag}\left(c_{1}, \ldots, c_{N}\right) \in \mathbb{R}_{+}^{N \times N}$ be a deterministic matrix with bounded spectral norm. Define $\bar{\delta}_{i}=\frac{1}{K} \operatorname{tr} \overline{\mathbf{D}}_{i} \overline{\mathbf{T}}(z, \mathbf{V}, \mathbf{0}), i=$
$1, \ldots, N$, where $\overline{\mathbf{T}}(z, \mathbf{V}, \mathbf{0})$ is given by Theorem 4 , and consider the matrix $\mathbf{R}(z, \mathbf{V}) \in \mathbb{C}^{N \times N}$, given as

$$
\mathbf{R}(z, \mathbf{V})=\operatorname{diag}\left(\frac{\bar{\delta}_{1}}{1+\bar{\delta}_{1}}, \ldots, \frac{\bar{\delta}_{N}}{1+\bar{\delta}_{N}}\right) .
$$

Then, for any $z \in \mathbb{C} \backslash \mathbb{R}_{+}$:

$$
\frac{1}{K} \operatorname{tr} \mathbf{C}\left[\mathbf{H}\left(\mathbf{H}^{\mathrm{H}} \mathbf{H}-z \mathbf{I}_{K}\right)^{-1} \mathbf{H}^{\mathbf{H}}-\mathbf{R}(z, \mathbf{V})\right] \underset{N, K \rightarrow \infty}{\stackrel{\text { a.s. }}{\longrightarrow}} 0 .
$$

Proof: Direct application of Lemmas 2, 3, 5, Theorem 4 and Lemma 1 together with the fact that $\frac{1}{K} \operatorname{tr} \overline{\mathbf{D}}_{i} \overline{\mathbf{T}}(z, \mathbf{V}, \mathbf{0}) \leq$ $\left\|\mathbf{D}_{i}\right\| /|z|$ leads to

$$
\begin{aligned}
& \frac{1}{K} \operatorname{tr} \mathbf{C H}\left(\mathbf{H}^{\mathrm{H}} \mathbf{H}-z \mathbf{I}_{K}\right)^{-1} \mathbf{H}^{\mathrm{H}} \\
\asymp & \frac{1}{K} \sum_{i=1}^{N} c_{i} \frac{\frac{1}{K} \operatorname{tr} \overline{\mathbf{D}}_{i} \overline{\mathbf{T}}(z, \mathbf{V}, \mathbf{0})}{1+\frac{1}{K} \operatorname{tr} \overline{\mathbf{D}}_{i} \overline{\mathbf{T}}(z, \mathbf{V}, \mathbf{0})} \\
= & \frac{1}{K} \operatorname{tr} \mathbf{C R}(z, \mathbf{V}) .
\end{aligned}
$$

Note that for $K<N$, the matrix $\mathbf{H}^{\mathrm{H}} \mathbf{H}$ has full rank with probability one and its inverse is therefore well defined. Although not proved, we argue that, similar to [16], the smallest eigenvalue of $\mathbf{H}^{\mathrm{H}} \mathbf{H}$ is almost surely uniformly bounded away from 0 as $N, K \rightarrow \infty$ while $\liminf \frac{N}{K}>1$, so that $\overline{\mathbf{T}}(0, \mathbf{V}, \mathbf{0})$ is well-defined and the convergence region can be extended to $z \in \mathbb{C} \backslash \mathbb{R}_{+} \cup\{0\}$. To give an intuition why this holds, write $\mathbf{H}^{\mathrm{H}} \mathbf{H}-\epsilon \mathbf{I}_{K}+\epsilon \mathbf{I}_{K}$ for some $\epsilon>0$, such that almost surely $\mathbf{H}^{\mathrm{H}} \mathbf{H}-\epsilon \mathbf{I}_{K}$ is a hermitian nonnegative matrix. This implies that $\frac{1}{K} \operatorname{tr}\left(\mathbf{H}^{\mathrm{H}} \mathbf{H}-\epsilon \mathbf{I}_{K}+\epsilon \mathbf{I}_{K}\right)^{-1} \leq \frac{1}{\epsilon}$. Using this bound, Theorem 4 can be extended to the case $z=0$.

Remark A.1: Moreover, we claim that for $\liminf \frac{N}{K}>1$, the convergence holds also for $z=0$.

Theorem 6: Assume $\liminf \frac{N}{K}>1$. Under the assumptions of Theorem 4, let $\mathbf{C}=\operatorname{diag}\left(c_{1}, \ldots, c_{N}\right) \in \mathbb{R}_{+}^{N \times N}, \mathbf{D}=$ $\operatorname{diag}\left(d_{1}, \ldots, d_{N}\right) \in \mathbb{R}_{+}^{N \times N}$ be deterministic matrices with bounded spectral norm. Define $\bar{\delta}_{i}=\frac{1}{K} \operatorname{tr} \overline{\mathbf{D}}_{i} \overline{\mathbf{T}}, i=1, \ldots, N$, where $\overline{\mathbf{T}}=\overline{\mathbf{T}}(0, \mathbf{V}, \mathbf{0})$ is given by Theorem 4 (see also Remark A.1). Let $\mathbf{v}=\left[v_{1}, \ldots, v_{N}\right]^{\top} \in \mathbb{C}^{N}$ and $\overline{\mathbf{J}} \in \mathbb{C}^{N \times N}$ which are respectively given as

$$
\begin{aligned}
{[\overline{\mathbf{J}}]_{k l} } & =\frac{\frac{1}{K} \operatorname{tr} \overline{\mathbf{D}}_{k} \overline{\mathbf{T}}_{l} \overline{\mathbf{T}}}{K\left(1+\bar{\delta}_{l}\right)^{2}} \\
v_{k} & =\sum_{l=1}^{N} \frac{\frac{1}{K} \operatorname{tr} \overline{\mathbf{D}}_{k} \overline{\mathbf{T}} d_{l} \overline{\mathbf{D}}_{l} \overline{\mathbf{T}}}{K\left(1+\bar{\delta}_{l}\right)} .
\end{aligned}
$$

Let the vector $\overline{\boldsymbol{\delta}}^{\prime}=\left[\bar{\delta}_{1}^{\prime}, \ldots, \bar{\delta}_{N}^{\prime}\right]^{\top} \in \mathbb{C}^{N}$ be defined as

$$
\overline{\boldsymbol{\delta}}^{\prime}=\left(\mathbf{I}_{N}-\overline{\mathbf{J}}\right)^{-1} \mathbf{v}
$$

and consider the matrix

$$
\mathbf{Q}(\mathbf{V}, \mathbf{D})=\operatorname{diag}\left(\frac{\bar{\delta}_{1}^{\prime}}{\left(1+\bar{\delta}_{1}\right)^{2}}, \ldots, \frac{\bar{\delta}_{N}^{\prime}}{\left(1+\bar{\delta}_{N}\right)^{2}}\right) .
$$


Denote $\mathbf{Q}=\mathbf{Q}(\mathbf{V}, \mathbf{D})$. Then

$$
\frac{1}{K} \operatorname{tr} \mathbf{C}\left[\mathbf{H}\left(\mathbf{H}^{\mathrm{H}} \mathbf{H}\right)^{-1} \mathbf{H}^{\mathrm{H}} \mathbf{D H}\left(\mathbf{H}^{\mathrm{H}} \mathbf{H}\right)^{-1} \mathbf{H}^{\mathrm{H}}-\mathbf{Q}\right] \underset{N, K \rightarrow \infty}{\stackrel{\text { a.s. }}{\longrightarrow} 0} \text {. }
$$

Proof: Consider the following function:

$$
f(x, z)=\frac{1}{K} \operatorname{tr} \mathbf{C H}\left(\mathbf{H}^{\mathrm{H}}\left(\mathbf{I}_{N}-x \mathbf{D}\right) \mathbf{H}-z \mathbf{I}_{K}\right)^{-1} \mathbf{H}^{\mathrm{H}}
$$

for $x<\inf \|\mathbf{D}\|^{-1}$. One can easily verify that its derivative $f_{x}^{\prime}(x, z)=\frac{d f(x, z)}{d x}$ evaluated at $x=z=0$ equals

$$
f_{x}^{\prime}(0,0)=\frac{1}{K} \operatorname{tr} \mathbf{C H}\left(\mathbf{H}^{\mathrm{H}} \mathbf{H}\right)^{-1} \mathbf{H}^{\mathrm{H}} \mathbf{D H}\left(\mathbf{H}^{\mathrm{H}} \mathbf{H}\right)^{-1} \mathbf{H}^{\mathrm{H}} .
$$

It remains now to find a deterministic equivalent of $f_{x}^{\prime}(0,0)$. First notice that for $K<N$ the matrix $\mathbf{H}^{\mathrm{H}} \mathbf{H}$ has full rank with probability one and $f(0,0)$ as well as $f_{x}^{\prime}(0,0)$ are consequently well defined. A straight-forward application of Theorem 5 leads to

$$
f(x, z)-\frac{1}{K} \operatorname{tr} \mathbf{C R}\left(z,\left(\mathbf{I}_{N}-x \mathbf{D}\right) \mathbf{V}\right) \underset{N, K \rightarrow \infty}{\stackrel{\text { a.s. }}{\longrightarrow}} 0 .
$$

In particular, we have $f(0,0) \asymp \frac{1}{K} \operatorname{tr} \mathbf{C R}(0, \mathbf{V})$ (see $\mathrm{Re}$ mark A.1). One can show that both functions $f(x, z)$ and $\bar{f}(x, z)=\frac{1}{K} \operatorname{tr} \mathbf{C R}\left(z,\left(\mathbf{I}_{N}-x \mathbf{D}\right) \mathbf{V}\right)$ are Stieltjes transforms of finite measures over $\mathbb{R}_{+}$which we denote by $\mu$ and $\bar{\mu}$, respectively. Theorem 5 implies that $\mu-\bar{\mu} \Rightarrow 0$ almost surely (see also [21, Proposition 2.2]). Further notice that the derivative $f_{x}^{\prime}(x, z)$ can be written under the form $\int F(\lambda, z) \mu(d \lambda)$, where $F(x, z)$ is a bounded continuous function. An application of the bounded convergence theorem ensures that $\int F(\lambda, z) \mu(d \lambda)-\int F(\bar{\lambda}, z) \bar{\mu}(d \bar{\lambda}) \rightarrow 0$ almost surely. This implies that $\bar{f}_{x}^{\prime}(x, z)=\frac{d}{d x} \frac{1}{K} \operatorname{tr} \mathbf{C R}\left(z,\left(\mathbf{I}_{N}-x \mathbf{D}\right) \mathbf{V}\right)$ is a deterministic equivalent of $f_{x}^{\prime}(x, z)$. Therefore,

$$
f_{x}^{\prime}(0,0)-\frac{1}{K} \operatorname{tr} \mathbf{C Q}(\mathbf{V}, \mathbf{D}) \underset{N, K \rightarrow \infty}{\stackrel{\text { a.s. }}{\longrightarrow}} 0
$$

where $\mathbf{Q}(\mathbf{V}, \mathbf{D})=\left.\frac{d}{d x} \mathbf{R}\left(0,\left(\mathbf{I}_{N}-x \mathbf{D}\right) \mathbf{V}\right)\right|_{x=0}$. Now,

$$
\mathbf{Q}(\mathbf{V}, \mathbf{D})=\operatorname{diag}\left(\frac{\bar{\delta}_{1}^{\prime}}{\left(1+\bar{\delta}_{1}\right)^{2}}, \ldots, \frac{\bar{\delta}_{N}^{\prime}}{\left(1+\bar{\delta}_{N}\right)^{2}}\right)
$$

where $\bar{\delta}_{k}^{\prime}=\frac{d \bar{\delta}_{k}}{d x}=\left.\frac{d}{d x} \frac{1}{K} \operatorname{tr} \overline{\mathbf{D}}_{k} \overline{\mathbf{T}}(0,(\mathbf{I}-x \mathbf{D}) \mathbf{V})\right|_{x=0}$ can be calculated as $(1 \leq k \leq N)$ :

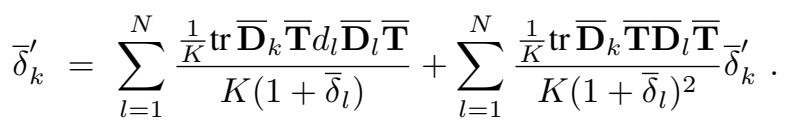

With the above definitions of $\overline{\boldsymbol{\delta}}^{\prime}$ and $\overline{\mathbf{J}}$, the last equation can be written in matrix form. By [20, Claim 1] (see also [22, Theorem 3.1]), we have $0<\operatorname{det}\left(\mathbf{I}_{N}-\overline{\mathbf{J}}\right)<\infty$ which implies that $\mathbf{I}_{N}-\overline{\mathbf{J}}$ has full rank and is thus invertible. Therefore, $\overline{\boldsymbol{\delta}}^{\prime}$ and, hence, $\mathbf{Q}(\mathbf{V}, \mathbf{D})$ are uniquely defined. Similarly to the proof of Theorem 5 we argue that [20, Claim 1] can be shown to hold for $z=0$ since the smallest eigenvalue of the matrix $\mathbf{H}^{\mathrm{H}} \mathbf{H}$ is almost surely uniformly bounded away from zero. This concludes the proof.

\section{REFERENCES}

[1] M. K. Karakayali, G. J. Foschini, and R. A. Valenzuela, "Network Coordination for Spectrally Efficient Communications in Cellular Systems," IEEE Wireless Commun. Mag., vol. 13, no. 4, pp. 56-61, Aug. 2006.

[2] T. Cover and J. A. Thomas, Elements of Information Theory, 2nd ed. John Wiley \& Sons, Inc., 2006.

[3] H. Weingarten, Y. Steinberg, and S. Shamai, "The Capacity Region of the Gaussian Multiple-Input Multiple-Output Broadcast Channel," IEEE Trans. Inf. Theory, vol. 52, no. 9, pp. 3936-3964, Sep. 2006.

[4] D. Gesbert, S. Hanly, H. Huang, S. Shamai, O. Simeone, and W. Yu, "Multi-Cell MIMO Cooperative Networks: A New Look at Interference," IEEE J. Sel. Areas Commun., vol. 28, no. 9, Dec. 2010.

[5] D. N. C. Tse and S. Hanly, "Linear Multiuser Receivers: Effective Interference, Effective Bandwidth and User Capacity," IEEE Trans. Inf. Theory, vol. 45, no. 2, pp. 641-657, Mar. 1999.

[6] S. Verdú and S. Shamai, "Spectral Efficiency of CDMA with Random Spreading," IEEE Trans. Inf. Theory, vol. 45, no. 2, pp. 622-640, Mar. 1999.

[7] D. N. C. Tse and O. Zeitouni, "Linear Multiuser Receivers in Random Environments," IEEE Trans. Inf. Theory, vol. 46, no. 1, pp. 171-188, Jan. 2000.

[8] J. Evans and D. N. C. Tse, "Large System Performance of Linear Multiuser Receivers in Multipath Fading Channels," IEEE Trans. Inf. Theory, vol. 46, no. 6, pp. 2059-2078, Sep. 2000.

[9] J.-M. Chaufray, W. Hachem, and P. Loubaton, "Asymptotic Analysis of Optimum and Suboptimum CDMA Downlink MMSE Receivers," IEEE Trans. Inf. Theory, vol. 50, no. 11, pp. 2620-2638, Nov. 2004.

[10] A. M. Tulino, L. Li, and S. Verdú, "Spectral Efficiency of Multicarrier CDMA," IEEE Trans. Inf. Theory, vol. 51, no. 2, pp. 479-505, Feb. 2005.

[11] P. Li, D. Paul, R. Narasimhan, and J. Cioffi, "On the Distribution of SINR for the MMSE MIMO Receiver and Performance Analysis," IEEE Trans. Inf. Theory, vol. 52, no. 1, pp. 271-286, Jan. 2006.

[12] Y.-C. Liang, G. Pan, and Z. D. Bai, "Asymptotic Performance of MMSE Receivers for Large Systems Using Random Matrix Theory," IEEE Trans. Inf. Theory, vol. 53, no. 11, pp. 4173-4190, Nov. 2007.

[13] A. Kammoun, M. Kharouf, W. Hachem, and J. Najim, "A Central Limit Theorem for the SINR at the LMMSE Estimator Output for LargeDimensional Signals," IEEE Trans. Inf. Theory, vol. 55, no. 11, pp. 5048-5063, 2009.

[14] K. R. Kumar, G. Caire, and A. Moustakas, "Asymptotic Performance of Linear Receivers in MIMO Fading Channels," IEEE Trans. Inf. Theory, vol. 55 , no. 10 , pp. 4398-4418, 2009.

[15] S. Verdú, Multiuser Detection. Cambridge University Press, New York, 1998.

[16] Z. D. Bai and J. W. Silverstein, "No Eigenvalues Outside the Support of the Limiting Spectral Distribution of Large Dimensional Sample Covariance Matrices," Annals of Probability, vol. 26, no. 1, pp. 316-345, Jan. 1998

[17] M. J. M. Peacock, I. B. Collings, and M. L. Honig, "Eigenvalue Distributions of Sums and Products of Large Random Matrices via Incremental Matrix Expansions," IEEE Trans. Inf. Theory, vol. 54, no. 5, pp. 2123-2138, 2008.

[18] J. W. Silverstein and Z. D. Bai, "On the Empirical Distribution of Eigenvalues of a Class of Large Dimensional Random Matrices," Journal of Multivariate Analysis, vol. 54, no. 2, pp. 175-192, 1995.

[19] R. Couillet and M. Debbah, Random Matrix Methods for Wireless Communications. Cambridge University Press, New York, to appear.

[20] J. Hoydis, J. Najim, R. Couillet, and M. Debbah, "Fluctuations of the Mutual Information in Large Distributed Antenna Systems with Colored Noise," in Forty-Eighth Annual Allerton Conference on Communication, Control, and Computing, Illinois, US, Sep. 2010.

[21] W. Hachem, P. Loubaton, and J. Najim, "Deterministic Equivalents for Certain Functionals of Large Random Matrices," Annals of Applied Probability, vol. 17, no. 3, pp. 875-930, 2007.

[22] _ "A CLT for Information-Theoretic Statistics of Gram Random Matrices with a Given Variance Profile," Annals of Applied Probability, vol. 18 , no. 6 , pp. 2071-2130, 2008. 